\title{
Malformações congênitas do sistema nervoso como causa de mortalidade infantil no estado do Pará no período de 2007 a 2016
}

\author{
Congenital malformations of the nervous system as a cause of child \\ mortality in the state of Pará in the period 2007 to 2016
}

Dilma Costa de Oliveira Neves ${ }^{1}$, Angela Regina Rosa Correa Pereira', Lorena Oliveira Silva de Melo', Melissa Nunes Leandro'

Resumo Objetivo: Analisar a ocorrência das malformações congênitas do sistema nervoso como causa de mortalidade infantil no Estado do Pará no período de 2007 a 2016. Método: Estudo descritivo-quantitativo-ecológico realizado no Departamento de Epidemiologia da Secretaria de Saúde do Estado do Pará. Foram incluídos os registros de crianças nascidas de mães residentes no Estado do Pará e que faleceram com menos de 1 ano, tendo como causa básica ou consequencial a malformação congênita do sistema nervoso. Resultados: Os óbitos por malformações congênitas, deformidades e anomalias cromossômicas representaram 17,3\% (4.219) do total dos óbitos em menores de 1 ano e dentre estes aqueles decorrentes das malformações congênitas do sistema nervoso constituem 21,3\% (900). A taxa média de mortalidade infantil para o período foi de 17,2 óbitos em cada 1000 nascidos vivos e a taxa por malformações congênitas do sistema nervoso contribuiu com aproximadamente 3,5\%. Dentre as principais malformações congênitas do sistema nervoso encontrou-se a hidrocefalia congênita (32,5\%), anencefalia e malformações similares $(25,9 \%)$, outras malformações congênitas do cérebro $(17,2 \%)$, e a microcefalia $(6,4 \%)$. A sobrevida dessas crianças no primeiro ano de vida foi 10,2\%. Conclusão: A análise das características do obituário por malformações congênitas do sistema nervoso no Estado do Pará permite identificar a fragilidade do planejamento reprodutivo e do pré-natal na prevenção dessas malformações, assim como a necessidade de implementar cuidados que possibilitem uma maior sobrevida dessas crianças e o apoio tanto para elas quanto para seus familiares.

Descritores: malformações congênitas; sistema nervoso; mortalidade infantil.

Summary Purpose: To analyze the occurrence of congenital malformations of the nervous system as a cause of infant mortality in the State of Pará in the period from 2007 to 2016. Method: The descriptive-quantitative-ecological study carried out in the Department of Epidemiology of the Health Department of the State of Pará. The records of children born to mothers residing in the State of Pará who died less than 1 year were included, having as basic or consequential cause the malformation congenital nervous system. Results: Deaths from congenital malformations, deformities and chromosomal anomalies represented $17.3 \%(4,219)$ of the total number of deaths in children under 1 year of age, and among them, those resulting from congenital malformations of the nervous system constitute $21.3 \%$ (900). The average mortality rate in for the period was 17.2 deaths per 1000 live births and the rate for congenital malformations of the nervous system contributed with approximately $3.5 \%$. Among the main congenital malformations of the nervous system were congenital hydrocephalus (32.5\%), anencephaly and similar malformations (25.9\%), other congenital malformations of the brain $(17.2 \%)$, and microcephaly $(6.4 \%)$. The survival of these children in the first year of life was $10.2 \%$. Conclusion: The analysis of the obituary characteristics by congenital malformations of the nervous system in the State of Pará allows us to identify the fragility of the reproductive and prenatal planning in the prevention of these malformations, as well as the need to implement care that allows a greater survival of these children and support for them and their families.

Keywords: congenital malformations; nervous system; child mortality.

${ }^{1}$ Centro Universitário do Estado do Pará - CESUPA, Belém, PA, Brasil

Fonte de financiamento: Nenhuma.

Conflito de interesses: Os autores declaram não haver conflitos de interesse.

Recebido: Junho 04, 2018

Aceito: Junho 28, 2018

Trabalho realizado no Centro Universitário do Estado do Pará - CESUPA, Belém, PA, Brasil.

Copyright Neves et al. Este é um artigo publicado em acesso aberto (Open Access) sob a licença Creative Commons Attribution, que permite uso, distribuição e reprodução em qualquer meio, sem restrições desde que o trabalho original seja corretamente citado. 


\section{Introdução}

A malformação congênita (MFC) é definida pela Organização Panamericana da Saúde (OPAS) como toda anomalia funcional ou estrutural no desenvolvimento do feto, decorrente de fatores originados antes do nascimento, sejam esses genéticos, ambientais ou desconhecidos. Ainda que o defeito não seja aparente a manifestação clínica mais tardia também é considerada como malformação congênita ${ }^{1}$.

A incidência destas encontra-se entre $2 \%$ e $3 \%$ dos nascidos vivos, e os principais fatores envolvidos são as condições hereditárias (genéticas), exposição a substâncias (medicamentos, álcool e drogas ilícitas), infecções (citomegalovirose, rubéola e toxoplasmose) e radiações, sendo que na maioria das vezes os fatores são desconhecidos ${ }^{2,3}$.

Dentre as malformações, as mais frequentes são as do sistema nervoso central (principalmente hidrocefalia e meningomielocele), as do sistema muscular (principalmente gastrosquise e onfalocele) e as cardiopatias ${ }^{4,5}$.

Em países cuja mortalidade infantil é elevada, as principais causas de morte no primeiro ano de vida relacionam-se, sobretudo, à desnutrição e às doenças infecciosas, e as malformações respondem por 5\% ou menos destas mortes ${ }^{6}$.

No Brasil, as MFC constituem a segunda causa de mortalidade infantil, determinando $11,2 \%$ destas mortes e a terceira na mortalidade de menores de cinco anos, representando 10,5\% destas. No município do Rio de Janeiro, no ano 2000, as MFC foram responsáveis por 15\% dos óbitos em menores de um ano, sendo a segunda causa no primeiro ano de vida. A metade (52\%) desses óbitos ocorreu no período neonatal precoce, $18 \%$ no período neonatal tardio e o restante antes de um ano de vida. Dentre as principais malformações congênitas foram observadas as anomalias do aparelho circulatório e do sistema nervoso central responsáveis por $58 \%$ destes óbitos 7 .

Em decorrência do aumento inesperado do diagnóstico de microcefalia relacionados a infecção materna pelo Zika vírus em nove estados brasileiros, incluindo o estado do Pará, tendo o primeiro caso detectado em maio de 2015, foi declarado o estado de emergência nacional de saúde visando o controle da epidemia ${ }^{8}$.

Nesse contexto o presente estudo teve por objetivo identificar o perfil das MFC do sistema nervoso e analisar a sua contribuição para a mortalidade infantil no Estado do Pará no período de 2007 a 2016.

\section{Método}

O estudo do tipo quantitativo-descritivo-ecológico foi aprovado pelo Comitê de Ética em Pesquisa em Seres Humanos do Centro Universitário do Estado do Pará sob processo de número 1.563.111 em 15/04/2016.

As variáveis analisadas foram data de nascimento, sexo, raça/cor, peso ao nascer, data do óbito, causa básica e consequencial do óbito.

Como MFC do Sistema Nervoso foi atendida a classificação estabelecida na X Revisão da Classificação Estatística Internacional de Doenças e Problemas Relacionados à Saúde (CID10). O sexo, raça/cor e peso ao nascer foram as variáveis utilizadas para a descrição do perfil do recém-nascido.

Nesta pesquisa foram incluídas as crianças nascidas vivas, de mães residentes no Estado do Pará, que evoluíram para o óbito com menos de 1 ano de idade, tendo como causa básica ou consequencial aMFC do sistema nervoso.

Os dados foram consolidados sob a forma de coeficientes e proporções. A análise estatística foi efetuada pelo teste estatístico Qui-Quadrado com auxílio do programa BioEstat 5.3. Foi aceito como diferença estatística significativa o valor de $p \leq 0,05$ para um nível de significância $\alpha=5 \%$.

\section{Resultados}

Os óbitos por MFC, deformidades e anomalias cromossômicas representaram 17,3\% (4.219) do total dos óbitos em menores de 1 ano, no período de 2007 a 2016 no estado do Pará e dentre estes os decorrentes das MFC do sistema nervoso central (SNC) constituem 21,3\% (900). As proporções de óbitos em menores de 1 ano por esses grupos de causas mantiveram-se semelhantes em todo o período (Tabela 1).

Observa-se, na Tabela 2, que as taxas de mortalidade infantil geral, na série histórica apresentaram valores semelhantes ( $p=0,9999)$, o mesmo ocorrendo na mortalidade infantil por MFC do sistema nervoso com valores estáveis no período. A taxa média de mortalidade infantil para o período foi de 17,2 óbitos /1000 nascidos vivos (NV) e a taxa por MFC do sistema nervoso contribuiu com aproximadamente 3,5\% $(0,6 / 17,2)$. 
Tabela 1. Distribuição dos óbitos por malformações congênitas, deformidades e anomalias cromossômicas e por malformações congênitas do sistema nervoso em crianças menores de um ano de mães residentes no Pará, ocorridos no período de 2007 a 2016

\begin{tabular}{|c|c|c|c|c|c|}
\hline Anos & $\begin{array}{c}\text { Total de } \\
\text { Óbitos }<1 \text { ano (A) }\end{array}$ & $\begin{array}{c}\text { Malformações Congênitas, } \\
\text { Deformidadese Anomalias } \\
\text { Cromossômicas (B) }\end{array}$ & $\%(B / A)^{*}{ }_{1}$ & $\begin{array}{c}\text { Malformações Congênitas do } \\
\text { Sistema Nervoso (C) }\end{array}$ & $\%(\mathrm{C} / \mathrm{B})^{*}{ }_{2}$ \\
\hline 2007 & 2814 & 396 & 14,1 & 101 & 25,5 \\
\hline 2008 & 2751 & 423 & 15,4 & 107 & 25,3 \\
\hline 2009 & 2619 & 416 & 15,9 & 94 & 22,6 \\
\hline 2010 & 2530 & 425 & 16,8 & 83 & 19,5 \\
\hline 2011 & 2471 & 442 & 17,9 & 92 & 20,8 \\
\hline 2012 & 2349 & 401 & 17,1 & 86 & 21,4 \\
\hline 2013 & 2295 & 433 & 18,9 & 78 & 18,0 \\
\hline 2014 & 2268 & 458 & 20,2 & 80 & 17,5 \\
\hline 2015 & 2164 & 396 & 18,3 & 77 & 19,4 \\
\hline 2016 & 2133 & 429 & 20,1 & 102 & 23,8 \\
\hline Total & 24394 & 4219 & 17,3 & 900 & 21,3 \\
\hline
\end{tabular}

${ }_{1}^{*}$ p-valor $=0,4577$ e. ${ }_{2}^{*}$ p-valor $=0,2314$ (Teste Qui-Quadrado de aderência).

Fonte: Sistema de Informação de Mortalidade - SIM, Departamento de Epidemiologia, Secretaria de Saúde do Estado do Pará - SESPA.

Tabela 2. Distribuição das taxas de mortalidade infantil geral; por malformações congênitas, deformidades e anomalias cromossômicas e por malformações congênitas do sistema nervoso em crianças menores de um ano de mães residentes no Pará, ocorridos no período de 2007 a 2016

\begin{tabular}{|c|c|c|c|c|c|}
\hline Anos & $\begin{array}{l}\text { Nascidos } \\
\text { Vivos (A) }\end{array}$ & $\begin{array}{c}\text { Total de } \\
\text { Óbitos }<1 \text { ano (B) }\end{array}$ & $\begin{array}{l}\text { Mortalidade Infantil } \\
\qquad(\mathrm{B} / \mathrm{A})^{*}\end{array}$ & $\begin{array}{l}\text { Malformações Congênitas do } \\
\text { Sistema Nervoso (C) }\end{array}$ & $\begin{array}{l}\text { Mortalidade } \\
\text { Infantil (C/A) }\end{array}$ \\
\hline 2007 & 150147 & 2814 & 18,7 & 101 & 0,7 \\
\hline 2008 & 151262 & 2751 & 18,2 & 107 & 0,7 \\
\hline 2009 & 143140 & 2619 & 18,3 & 94 & 0,7 \\
\hline 2010 & 140687 & 2530 & 18,0 & 83 & 0,6 \\
\hline 2011 & 141974 & 2471 & 17,4 & 92 & 0,6 \\
\hline 2012 & 137837 & 2349 & 17,0 & 86 & 0,6 \\
\hline 2013 & 139416 & 2295 & 16,5 & 78 & 0,6 \\
\hline 2014 & 143503 & 2268 & 15,8 & 80 & 0,6 \\
\hline 2015 & 132213 & 2164 & 16,4 & 77 & 0,6 \\
\hline 2016 & 135130 & 2133 & 15,8 & 102 & 0,8 \\
\hline $\begin{array}{l}\text { Média para o } \\
\text { período }\end{array}$ & 139906 & 2410 & $17,2 \pm 1,1$ & $89 \pm 10,8$ & 0,6 \\
\hline
\end{tabular}

${ }_{1}^{*}$ p-valor=0,9999 (Teste Qui-Quadrado de aderência).

Fonte: Sistema de Informação de Mortalidade - SIM, Departamento de Epidemiologia, Secretaria de Saúde do Estado do Pará - SESPA.

As principais MFC do sistema nervoso foram a hidrocefalia congênita (32,5\%), anencefalia e malformações similares $(25,9 \%)$ e outras malformações congênitas do cérebro $(17,2 \%)$. Observa-se que todos os óbitos por anencefalia ocorreram antes do primeiro ano de vida. A microcefalia foi a quarta causa $(6,4 \%)$ de óbito infantil encontrada nos registros de óbitos em crianças menores de 1 ano (Tabela 3).

Na Figura 1 observa-se uma redução na proporção dos óbitos por hidrocefalia congênita e aumento por microcefalia em menores de um ano a cada ano do período.

A probabilidade para uma criança que nasce com malformação congênita do sistema nervoso sobreviver ao final do primeiro ano de vida foi de 10,2\%. (Figura 2).

Quanto às características dos recém-nascidos observa-se a maioria do sexo feminino $(50,2 \%)$, com peso baixo ao nascer $(31,9 \%)$ e de raça/cor parda $(73,7 \%)$. (Tabela 4$)$. 
Tabela 3. Distribuição das malformações congênitas do sistema nervoso segundo os subgrupos alfanuméricos (Q00-Q07) da CID-10, Pará, 2006 a 2015

\begin{tabular}{|c|c|c|c|c|c|c|}
\hline \multirow{2}{*}{ Malformações congênitas do Sistema Nervoso } & \multicolumn{2}{|c|}{ Óbito $<1$ ano } & \multicolumn{2}{|c|}{ Óbito $\geq 1$ ano } & \multicolumn{2}{|c|}{ Total de Óbitos } \\
\hline & Frequência & $\%$ & Frequência & $\%$ & Frequência & $\%$ \\
\hline Hidrocefalia congênita & 259 & 28,8 & 107 & 47,6 & 366 & 32,5 \\
\hline Anencefalia e malformações similares & 292 & 32,4 & - & 0,0 & 292 & 26,0 \\
\hline Outras malformações congênitas do cérebro* ${ }_{1}^{*}$ & 164 & 18,2 & 30 & 13,3 & 194 & 17,2 \\
\hline Microcefalia & 51 & 5,7 & 21 & 9,3 & 72 & 6,4 \\
\hline Espinha bífida & 62 & 6,9 & 7 & 3,1 & 69 & 6,1 \\
\hline Encefalocele & 39 & 4,3 & 18 & 8,0 & 57 & 5,1 \\
\hline Outras malformações congênitas do sistema nervoso ${ }_{2}^{*}$ & 25 & 2,8 & 14 & 6,2 & 39 & 3,5 \\
\hline Outras malformações congênitas da medula espinhal ${ }_{3}^{*}$ & 8 & 0,9 & 28 & 12,4 & 36 & 3,2 \\
\hline Total & 900 & 100,0 & 225 & 100,0 & 1125 & 100,0 \\
\hline
\end{tabular}

* Malformação congênita não especificada do encéfalo (61); Outras deformidades por redução do encéfalo (102); Malformações congênitas do corpo caloso (11); Outras malformações congênitas especificadas do encélalo (11); Holoprosencefalia (9). ${ }_{2}^{*}$ Malformação congênita não especificada do sistema nervoso (24); Sindrome de Arnold Chiari (11); Outras malformações congênitas especificadas do sistema nervoso (5). ${ }_{3}^{*}$ Hipoplasia e displasia da medula espinhal (20); Malformação congênita não especificada da medula espinal (7); Malformação congênita não especificada da medula espinhal (5); Amielia (4).

Fonte: SIM-Sistema de Informação de Mortalidade-Departamento de Epidemiologia- SESPA- Secretaria de Saúde do Estado do Pará.

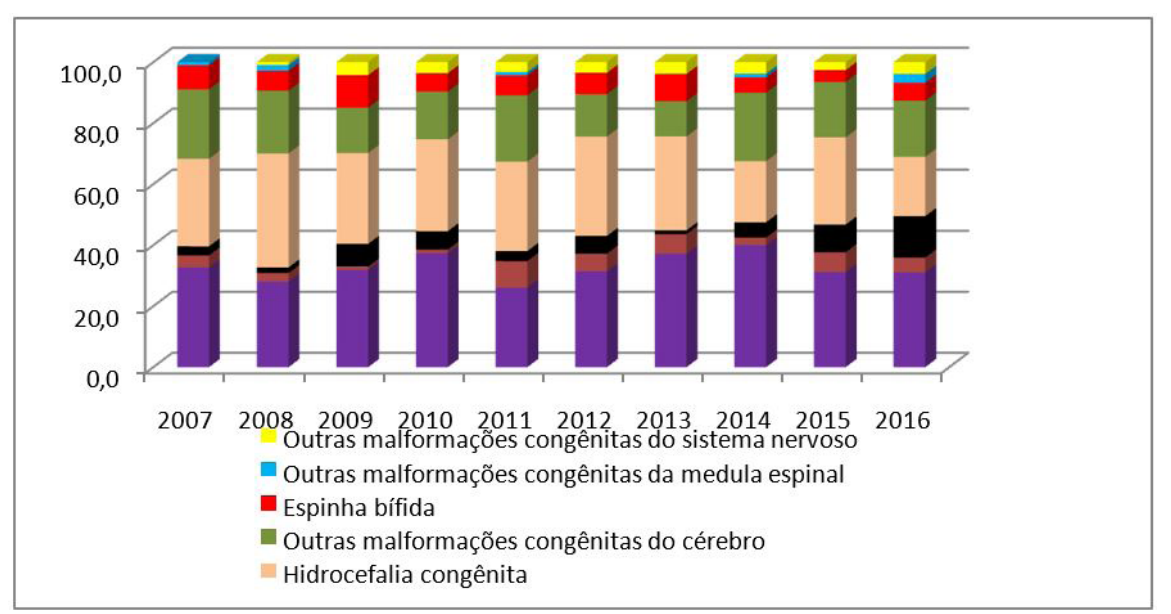

Figura 1. Distribuição dos óbitos infantis por malformações congênitas do sistema nervoso segundo os anos do período, Pará, 2007 a 2016. Fonte: SIM - Sistema de Informação de Mortalidade - Departamento de Epidemiologia SESPA Secretaria de Saúde do Estado do Pará.

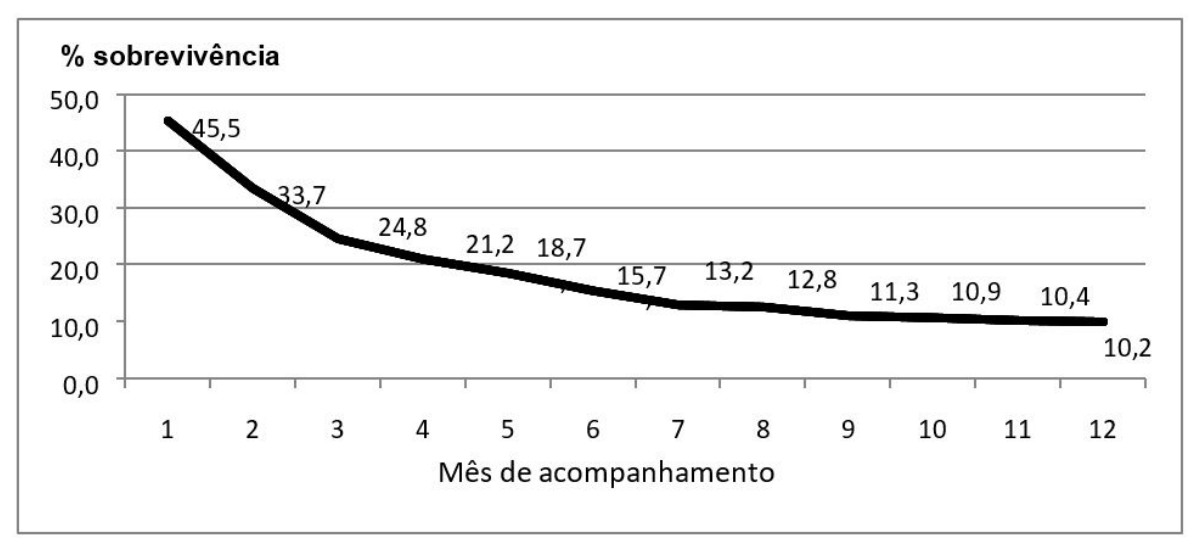

Figura 2. Sobrevida até o décimo segundo mês de vida dos recém-nascidos com malformação congênita do sistema nervoso, Pará, 2007 a 2016.

Fonte: SIM - Sistema de Informação de Mortalidade - Departamento de Epidemiologia - SESPA - Secretaria de Saúde do Estado do Pará. 
Tabela 4. Perfil dos recém-nascidos com malformação congênita do sistema nervoso, Pará, 2007 a 2016

\begin{tabular}{|c|c|c|}
\hline Características do Recém Nascido & $N=900$ & $\%$ \\
\hline \multicolumn{3}{|l|}{ Sexo } \\
\hline Masculino & 452 & 50,2 \\
\hline Feminino & 440 & 48,9 \\
\hline Ignorado & 8 & 0,9 \\
\hline \multicolumn{3}{|l|}{ Peso } \\
\hline$\geq 1000 \mathrm{~g}$ a $<2500 \mathrm{~g}$ & 287 & 31,9 \\
\hline$\geq 2500 \mathrm{~g} \mathrm{a}<3000 \mathrm{~g}$ & 183 & 20,3 \\
\hline$\geq 3000 \mathrm{~g}$ a $4000 \mathrm{~g}$ & 209 & 23,2 \\
\hline$>4000 \mathrm{~g}$ & 51 & 5,7 \\
\hline Ignorado & 170 & 18,9 \\
\hline \multicolumn{3}{|l|}{ Raça/cor } \\
\hline Parda & 663 & 73,7 \\
\hline Branca & 202 & 22,4 \\
\hline Indígena & 9 & 1,0 \\
\hline Preta & 7 & 0,8 \\
\hline Amarela & 1 & 0,1 \\
\hline Ignorada & 18 & 2,0 \\
\hline
\end{tabular}

Fonte: SIM-Sistema de Informação de Mortalidade-Departamento de Epidemiologia- SESPA- Secretaria de Saúde do Estado do Pará.

\section{Discussão}

Segundo o Ministério da Saúde (MS) a mortalidade infantil reduziu de 47,1, em 1990 para 15,6/1000 nascidos vivos (NV) em 2010 ${ }^{\circ}$. No entanto as proporções de óbitos em menores de 01 ano por MFC em geral mantiveram-se estáveis no período, assim como por MFC do sistema nervoso (Tabela 1). Esta proporção é semelhante a encontrada em estudo realizado em uma maternidade da cidade do Recife no período de 2004 a $2005^{10}$. Em relação a taxa de mortalidade infantil geral observou-se valores semelhantes $(\mathrm{p}=0,9999)$ entre os anos de 2007 e 2016, assim como os valores da mortalidade infantil por malformações do sistema nervoso central (SNC) que manteve uma estabilidade ao longo desse período (Tabela 2).

Um estudo sobre a estrutura das maternidades verificou que a disponibilidade de equipamentos essenciais e estratégicos para viabilizar a sobrevivência do recém-nascido nas emergências tem como locais de maior deficiência os hospitais do Norte/Nordeste. Assim, a desigualdade geográfica na oferta de serviços hospitalares do SUS indicam vazios assistenciais que impõem deslocamento geográfico para a internação para o parto, em um contexto de baixo suporte à assistência à gestante contribuindo para o aumento da mortalidade infantil ${ }^{11}$.

Em um estudo em Ribeirão Preto-SP ${ }^{12}$, entre os anos de 1991 e 2000, a análise das taxas de mortalidade mostrou um claro declínio na mortalidade fetal, neonatal precoce e perinatal, com uma diminuição mais significativa nas taxas de mortalidade infantil perinatal (de 56,4\% em 1992 para 19,3\% em 2000) o que estava fortemente relacionada com a melhoria do atendimento pré e neonatal. Isto em decorrência dos avanços nos cuidados obstétricos e neonatais, como a consolidação da ultrassonografia como método não invasivo de avaliação fetal, o uso de corticóides para aumentar a maturidade pulmonar fetal e reduzir as doenças respiratórias, e o uso de medicamentos mais efetivos para prevenir o parto prematuro e aumentar a taxa de sobrevida dos recém-nascidos. Além disso, uma maior ênfase dada aos cuidados pré-natais (testes de triagem e tratamento de doenças maternas e fetais), educação de gestantes e promoção da amamentação.

Entretanto, em um estudo no Rio de Janeiro ${ }^{13}$ a taxa de mortalidade infantil dos óbitos por causas não evitáveis, isto é, aquelas que não dispõem de tecnologia médica e sanitária capaz de evitar o óbito, foi igual a 4,8/ mil NV, uma taxa 2,9 vezes menor que a de mortalidade infantil geral (13,9/ mil NV).

A questão da redução da mortalidade neonatal foi assumida como uma das metas do MS para a redução das desigualdades regionais no País em 2009 com o objetivo traçado de reduzir em $5 \%$ as taxas de mortalidade neonatal nas regiões da Amazônia Legal e do Nordeste brasileiro. A mortalidade em crianças menores de 1 ano teve expressiva queda nas últimas décadas no Brasil, graças às estratégias implementadas pelo governo federal, 
como ações para diminuição da pobreza, ampliação da cobertura da Estratégia Saúde da Família, ampliação das taxas de aleitamento materno exclusivo, entre outras. Porém a meta de garantir o direito à vida e à saúde a toda criança brasileira ainda não foi alcançada, persistindo desigualdades regionais e sociais inaceitáveis?.

Como resultado de estudo realizado em uma maternidade na cidade de Aracaju envolvendo todos os nascimentos ocorridos entre janeiro de 2010 e dezembro de 2011 foram encontrados defeitos do fechamento do tubo neural, casos de mielomeningocele, de anencefalia e de encefalocele com frequências similares, sendo que em um caso houve associação entre mielomeningocele e anencefalia ${ }^{14}$.

Outro estudo realizado na Unidade de Neonatologia de um hospital na cidade de Caxias do Sul/RS, no período de março/1998 a junho/2008, mostrou que a hidrocefalia isolada foi observada em três casos $(9,4 \%)$; todos os RN acometidos eram nativivos e um dos casos apresentou-se como pequeno para a idade gestacional. No entanto a soma dos casos de hidrocefalia aos de meningomielocele faz com que a frequência das malformações encontradas seja de $25 \%{ }^{15}$. Enquanto que no estudo atual no estado do Pará observa-se que a hidrocefalia isolada foi registrada em $32,5 \%$ dos casos de MFC do SNC. Porém não houve registro da mesma associada a outras MFC (Tabela 3).

A ausência de registro de hidrocefalia associada a outras MFC do SNC pode ser decorrente de falhas no preenchimento do atestado de óbito, visto que outro estudo ${ }^{16}$ refere o encontro de óbitos tanto por hidrocefalia isolada quanto associada, além de estabelecer que a hidrocefalia isolada é a MFC do SNC mais incidente seguida daquela associada a outras MFC.

Um estudo realizado na Universidade Estadual de Londrina encontrou como idade mínima do óbito 97 dias e a máxima de 155,73 dias, sendo que a causa básica de morte para 86,4\% crianças foi ocasionada pela própria malformação ${ }^{17}$, o que difere do achado de $54,5 \%$ dos óbitos no primeiro mês de vida e dentre estes $100 \%$ por anencefalia o que contribui para uma sobrevida das crianças que nascem com MFC do SNC de apenas 10,2\% (Figuras 1 e 2).

Isso ocorre devido as diferenças nas estruturas hospitalares, haja vista que muitas instituições não possuem estrutura de suporte que possa fornecer cuidados adequados dentro e fora do ambiente hospitalar, como equipe multidisciplinar adequada, unidades de tratamento intensivo, dentre outros, que possam assim instituir a reabilitação e a prevenção de agravos. Reafirma-se, assim, o aspecto crônico das anomalias interferindo diretamente na qualidade de vida da criança e sua família. Ambos necessitam de cuidados contínuos que implicam altos custos, somados ao trauma psicológico familiar e às dificuldades de adaptação à sociedade ${ }^{18}$.

Com relação às características do recém-nascido, nota-se conformidade com o estudo da maternidade em Recife ${ }^{10}$, no qual a maioria deles eram prematuros, sendo cerca de $55 \%$ com malformações e desses $50,4 \%$ pesavam menos que 2500 g e a maioria do sexo feminino (53,1\%), concordando com o estudo atual, sendo $31,9 \%$ dos RN com malformações com peso $<2500$ g, porém com a maioria do sexo masculino $(50,2 \%)$ (Tabela 4). Corroborando com outro estudo, em que os autores encontraram que 50 (53,8\%) recém-nascidos eram do gênero masculino, $41(44,1 \%)$ do gênero feminino e $2(2,1 \%)$ de gênero indeterminado ${ }^{19}$.

O Ministério da Saúde (MS) em 2012 mostrou que a prematuridade é um fator de risco de suma importância na mortalidade infantil, e vem tendo um aumento da incidência da prematuridade e baixo peso ao nascer em capitais e cidades de maior porte do país, podendo estar associado à prevalência da realização das cesarianas 9 .

Há estudos que relacionam as alterações do sistema nervoso e prematuridade com o fumo na gravidez, sendo responsável por $20 \%$ dos casos de fetos com baixo peso ao nascer, $8 \%$ dos partos prematuros e $5 \%$ de todas as mortes perinatais. Há estudos que mostram que o tabagismo pode contribuir para a morte súbita do bebê, além de causar importantes alterações no sistema nervoso ${ }^{20}$. Mas de uma forma geral, a associação entre defeito congênito e nascimento pré-termo não está bem definida; todavia não se sabe se os defeitos congênitos teriam sido o fator causal da menor duração da gestação, indicando que a presença da malformação não levou, necessariamente, à prematuridade; ou seja, a deformidade não se mostrou como um fator de interrupção precoce da gravide ${ }^{21}$.

\section{Conclusão}

As limitações do estudo decorrentes da subnotificação e de falhas nos registros dos óbitos permite inferir que nem todas as crianças diagnosticadas com malformações estejam cadastradas no sistema de informação de mortalidade, havendo necessidade de um maior investimento do Estado para capacitação de médicos visando o adequado preenchimento do atestado de óbito. 
No entanto, a análise das características do obituário por malformações congênitas do sistema nervoso no Estado do Pará permite identificar a fragilidade do planejamento reprodutivo e do pré-natal na prevenção dessas malformações, assim como a necessidade de implementar cuidados que possibilitem uma maior sobrevida dessas crianças e o apoio tanto para elas quanto para seus familiares.

\section{Referências}

1. Organização Panamericana de Saúde. Saúde materno infantil: atenção primária nas Américas. Organização Panamericana de Saúde: Washington; 1994.

2. Jones KL. Dysmorphology. In: Berman RE, Kliegman RM, Jenson HB. Nelson textbook of pediatrics. 17th ed. Philadelphia: Saunders; 2004. p. 616-23.

3. Azevedo PF, Souza ASR, Noronha No C, Lima MMS, Cardoso AS, Porto AMF. Citomegalovirose congênita: relato de caso. Rev Bras Ginecol Obstet. 2005;27(12):750-8. http://dx.doi.org/10.1590/S0100-72032005001200008.

4. Souza JMP, Buchalla CM, Laurenti R. Estudo da morbidade e da mortalidade perinatal em maternidades. III - Anomalias congênitasem nascidos vivos. Rev Saude Publica. 1987;21(1):5-12. http://dx.doi.org/10.1590/S0034-89101987000100002.

5. Swain S, Agrawal A, Bhatia BD. Congenital malformations at birth. Indian Pediatr. 1994;31(10):1187-91. PMid:7875778.

6. World Health Organization. World health report. Geneva: WHO; 1998. p. 43-47.

7. Costa CMS. Perfil das malformações congênitas em uma amostra de nascimentos no município do Rio de Janeiro1999 - 2001. Rio de Janeiro: Fundação Oswaldo Cruz; 2005.

8. Campos GS, Bandeira AC, Sardi SI. Zika Virus Outbreak, Bahia, Brazil. Emerg Infect Dis. 2015;21(10):1885-6. http://dx.doi.org/10.3201/ eid2110.150847. PMid:26401719.

9. Brasil. Atenção à saúde do recém-nascido: guia para os profissionais de saúde - cuidados gerais. 2. ed. Brasilia: Ministério da Saúde; 2012.

10. Amorim MMM, Vilela PC, Santos ARV, Lima ALMV, Melo EFP, Bernardes HF, et al. Impacto das malformações congênitas na mortalidade perinatal e neonatal em uma maternidade-escola do Recife. Rev Bras Saude Mater Infant. 2006;6(Supl 1):S19-S25.

11. Bittencourt SDA, Reis LGC, Ramos MM, Rattner D, Rodrigues PL, Neves DCO, et al. Estrutura das maternidades: aspectos relevantes para a qualidade da atenção ao parto e nascimento. Cad Saúde Pública. 30(Sup): 208-219, 2014 http://dx.doi.org/10.1590/0102-311X00176913.

12. Duarte G, Coltro PS, Bedone RV, Nogueira AA, Gelonezzi GM, Franco LJ. Trends in the modes of delivery and their impact on perinatal mortality rates. Rev Saude Publica. 2004;38(3):379-84. http://dx.doi.org/10.1590/S0034-89102004000300007. PMid:15243667.

13. Noronha GA, Torres TG, Kale PL. Análise da sobrevida infantil segundo características maternas, da gestação, do parto e do recém-nascido na coorte de nascimento de 2005 no Município do Rio de Janeiro-RJ, Brasil. Epidemiol Serv Saúde. 2012;21(3):419-30. http://dx.doi. org/10.5123/S1679-49742012000300007.

14. Nazer H J, López-Camelo J, Castilla EE. ECLAMC: Estudio de 30 años de vigilância epidemiológica de defectos de tubo neural en Chile y em Latinoamérica. Rev Med Chil. 2001;129(5):531-9. http://dx.doi.org/10.4067/S0034-98872001000500009. PMid:11464535.

15. Pante FR, Madi JM, Araújo BF, Zatti H, Madi SRC, Rombaldi RL. Malformações congênitas do sistema nervoso central: prevalência e impacto perinatal. Revista da AMRIGS. 2011;55(4):339-44.

16. Noronha L, Medeiros F, Martins VD, Sampaio GA, Serapião MJ, Kastin G, et al. Malformations of the central nervous system: analysis of 157 pediatric autopsies. Arq Neuropsiquiatr. 2000;58(3B):890-6. http://dx.doi.org/10.1590/S0004-282X2000000500015. PMid:11018827.

17. Brito, ECG. Mortalidade infantil por anomalias congênitas: itinerário percorrido do adoecimento ao óbito [docência]. Londrina: Universidade Estadual de Londrina, Centro de Ciências da Saúde; 2015

18. Polita NB, Ferrari RAP, Moraes PS, Sant'Anna FL, Tacla MTGM. Anomalias congênitas: internações em unidade pediátrica, Congenital anomalies: hospitalization in a pediatricunit. Rev Paul Pediatr. 2013;31(2):205-10. http://dx.doi.org/10.1590/S0103-05822013000200011. PMid:23828057.

19. Pimenta MS, Calil VMLT, Krebs VLJ. Perfil das malformações congênitas no berçário anexo à maternidade do Hospital das Clínicas da Universidade de São Paulo. São Paulo. Rev Med. 2010;89(1):50-6.

20. Leopércio W, Gigliotti A. Tabagismo e suas peculiaridades durante a gestação: uma revisão crítica. Jornal Brasileiro de Pneumologia. 2004; 30(2):176-85.

21. Rodrigues LS, Lima RHS, Costa LC, Batista RFL. Características das crianças nascidas com malformações congênitas no município de São Luís, Maranhão, 2002-2011. Epidemiol Serv Saúde. 2014; 23(2):295-304. http://dx.doi.org/10.5123/S1679-49742014000200011. 


\section{Autor correspondente}

Lorena Oliveira Silva de Melo

Travessa Pirajá, 716

CEP 66095631, Belém, PA, Brasil

Tel.: (91) 98279-9821

E-mail: lorena_osm@hotmail.com

\section{Informação sobre os autores}

DCON é epidemiologista, MSc em clínica das doenças tropicais pela Instituição (UFPA).

ARRCP é especialista em Pediatria, docente do curso de Medicina da Instituição (CESUPA).

LOSM e MNL são graduandos do curso de Bacharelado em Medicina no Centro Universitário do Estado do Pará (CESUPA).

\section{Contribuição dos autores}

DCON foi a orientadora do trabalho; LOSM e MNL são autoras e pesquisadoras do trabalho.

Todos os autores leram e aprovaram a versão final submetida ao Pará Research Medical Journal. 